follow-up/ died). Perfusion index (Figure 1) varies significantly with time $(\mathrm{p}=0.0015)$. After adjustment for multiple testing, the increase from baseline is statistically significant at 6 weeks ( $p$ $=0.014)$ and approaches significance at 6 months $(\mathrm{p}=0.074)$ post treatment.

Conclusion Change in microvascular perfusion is most pronounced 6 weeks following the completion of treatment. This finding will be used in the design of future clinical studies, where measurement of MPI will provide a robust comparison of different existing and emerging treatment protocols for NSCLC with regard to their effects on myocardial physiology.

Funding Beatson Oncology Centre Fund

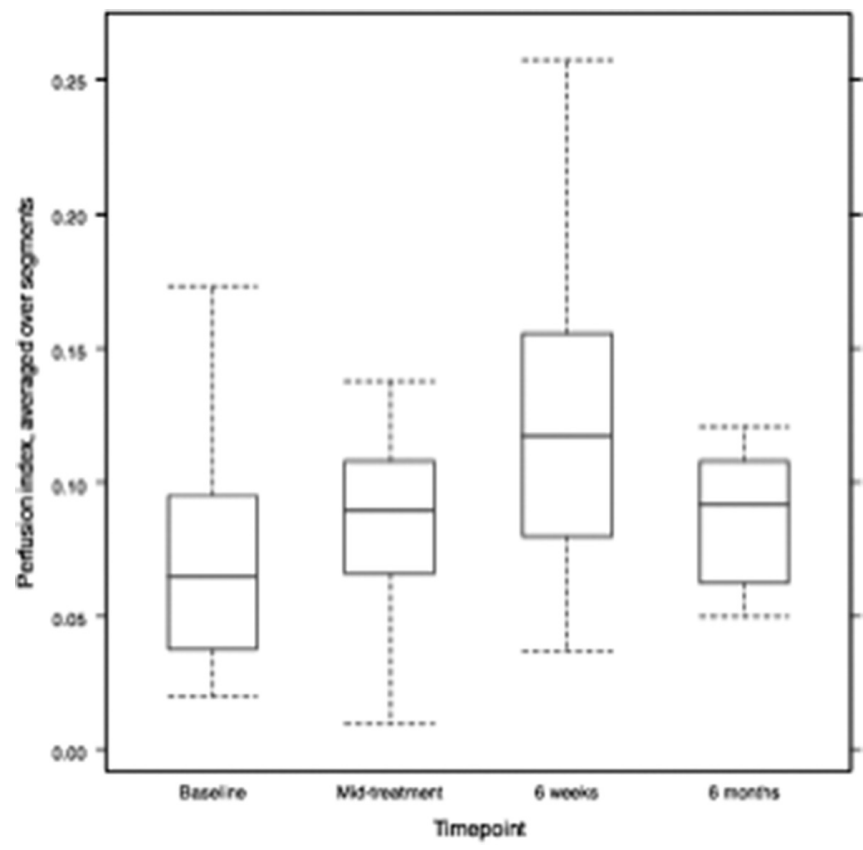

Abstract 16 Figure 1 Boxplots of global myocardial perfusion index values by time point

\section{A CASE-CONTROL STUDY WITH COMPUTATIONAL MODELLING OF ACUTE LEFT VENTRICULAR DYSFUNCTION}

${ }^{1} \mathrm{H}$ Gao, ${ }^{2,3, *} \mathrm{~K}$ Mangion, ${ }^{2,3} \mathrm{D}$ Carrick, ${ }^{1} \mathrm{X}$ Luo, ${ }^{2,3} \mathrm{C}$ Berry. ${ }^{1}$ School of Mathematics and Statistics, University of Glasgow, UK; ${ }^{2}$ Institute of Cardiovascular and Medical Science, University of Glasgow, UK; ${ }^{3}$ Golden Jubilee National Hospital, Clydebank, UK

\subsection{6/heartinl-2016-309668.17}

Introduction The pathophysiology of acute left ventricular dysfunction after acute ST-elevation myocardial infarction (STEMI) is incompletely understood. In this study, we carried out a casecontrol study of left ventricular (LV) biomechanical behaviour in vivo using an immersed boundary-finite element (IB/FE) method. Methods Cardiac magnetic resonance images were acquired from six healthy volunteers (the healthy group) and six patients with acute LV dysfunction post-acute STEMI (the MI group) with no-reflow, defined as an acute reduction in myocardial blood flow despite a patent epicardial coronary artery. The two groups were age matched. The passive and active myocardial parameters were determined from the measured in vivo data (LV cavity volume and strain) to match the LV dynamics in diastole and systole.

Results Student's t-test was used for significance test. Our results show that even though the average measured peak cuff-pressure in the MI group is much lower than the healthy group (122 \pm 21 vs. $150 \pm 20 \mathrm{mmHg}, \mathrm{p}<0.05)$, the peak systolic active tension in the MI group is higher $(68.9 \pm 6.8$ vs. $64.7 \pm 8.4 \mathrm{kPa}, \mathrm{p}$ $=0.15)$, therefore the required active tension per $\mathrm{mmHg}$ increase is significantly higher in the MI group $(0.57 \pm 0.05$ vs. $0.43 \pm 0.05 \mathrm{kPa} / \mathrm{mmHg}, \mathrm{p}<0.01)$, which may suggest that the viable myocardium in the MI group is working harder to compensate for the loss of contraction even with much lower blood pressure. Compared to the healthy group, the MI group also has a larger end-diastolic volume $(143 \pm 23$ vs. $122 \pm 16 \mathrm{~mL}, \mathrm{p}=$ $0.09)$, and a lower ejection fraction $(39 \pm 3 \%$ vs. $53 \pm 2 \%$, p $<0.01)$.

Conclusions In summary, we have shown for the first time that, compared with controls, patients with recent ST-elevation myocardial infarction exhibit increased active tension generation in combination with predictable changes in end-diastolic volume and ejection fraction. Active tension generation is a novel biomechanical index, and its clinical and prognostic significance merits further study.

Funding British Heart Foundation (PG/14/64/31043)

\section{CINE-DERIVED STRAIN USING THE GLASGOWHEART METHOD}

${ }^{1, *} \mathrm{~K}$ Mangion, ${ }^{2} \mathrm{H}$ Gao, ${ }^{1} \mathrm{~A}$ Radjenovic, ${ }^{2} \mathrm{X}$ Luo, ${ }^{3} \mathrm{C}$ Haig, ${ }^{3} \mathrm{C}$ Berry. ${ }^{1} \mathrm{BHF}$ Glasgow Cardiovascular Research Centre, University of Glasgow, Glasgow, UK; ${ }^{2}$ School of Mathematics and Statistics, University of Glasgow, Glasgow, UK; ${ }^{3}$ Robertson Centre for Biostatistics, University of Glasgow, Glasgow, UK

\subsection{6/heartjnl-2016-309668.18}

Background The GlasgowHeart method is designed to overcome some limitations of currently available feature-tracking methods by incorporating all of the myocardial tissues using an in-house developed intensity-based b-spline deformable registration method. The aim of this pilot study was to ensure that peak circumferential strain (Ecc) estimation is feasible and reproducible with minimal intra- and inter- observer variability.

Methods 20 healthy volunteers underwent 1.5T CMR twice, $<2$ days apart. Mid- LV cine sequences, were analysed with the Glasgowheart software (Figure 1). Two observers independently analysed 40 short axis slices for inter-observer variability. One observer reanalysed the 40 short axis slices. Pearson correlation and Bland-Altman analysis were used.

Results 20 participants were used in the analysis (mean age \pm SD 49.5 years (17.2) 50\% male). Ecc measured on the first set of MRIs by the two observers was highly correlated $(\mathrm{R}=0.915$, $\mathrm{p}<0.001$ ) and in excellent agreement (mean difference $=0.01$; 95\% LoA: -0.01, 0.02). Repeated image analysis also disclosed a high degree of association in paired measurements of Ecc that was strongly correlated $(\mathrm{R}=0.915, \mathrm{p}<0.001)$ and in excellent agreement $($ mean $=0.00 ; 95 \%$ LoA: $-0.02,0.01)$. Ecc measured in the second set of MRIs by 2 observers was well correlated $(\mathrm{R}=0.937, \mathrm{p}<0.001)$ and in excellent agreement (mean $=0.00 ; 95 \%$ LoA: -0.016 and 0.021$)$. The repeated image analysis at follow-up yielded Ecc that was well correlated $(\mathrm{R}=0.942, \mathrm{p}<0.001)$ and in excellent agreement (mean $=$ 0.00; 95\% LoA: -0.009 and 0.009). There was no difference between the average global Ecc at different time points $(p>$ $0.05)$. 

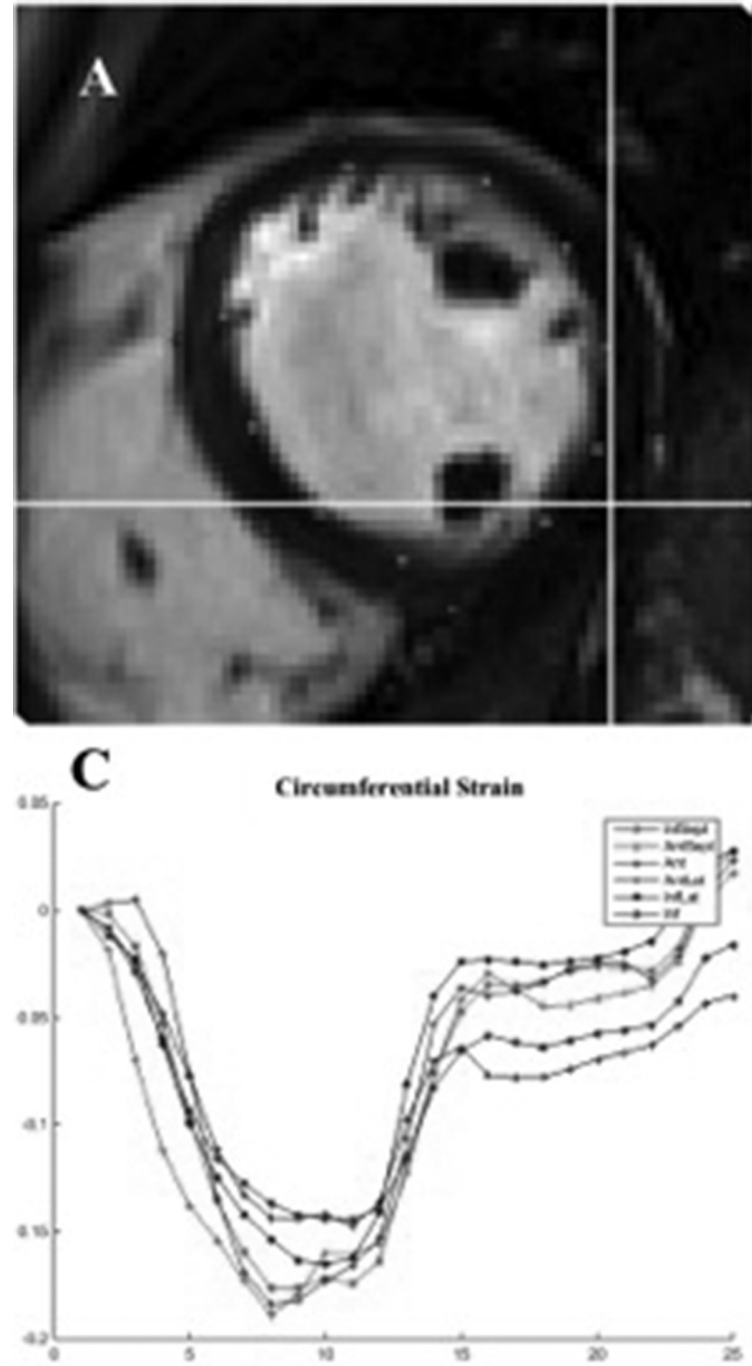

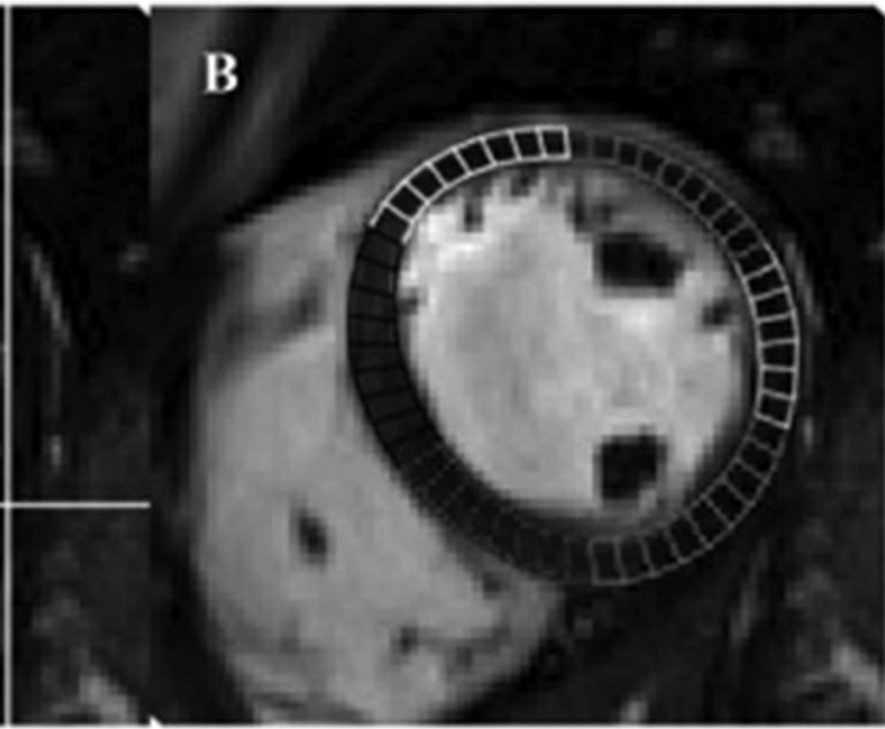

D

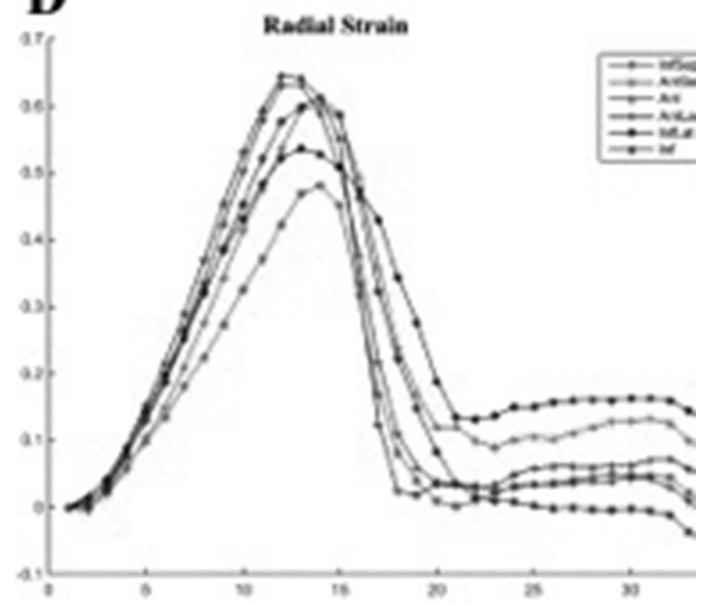

Abstract 18 Figure 1 The Glasgowheart pixel-tracking software. (A) shows border delineation (B) shows automatic segmentation using the inferior right ventricular insertion point. (C) and (D) show graphical output as circumferential and radial strain graphs, respectively

Conclusion The GlasgowHeart method is a robust and reproducible method of assessing cine-derived circumferential strain. By tracking a higher proportion of voxels than the currently available feature tracking software, it has clear potential to provide a more accurate assessment of strain.

\section{FEASIBILITY OF NATIVE HIGH-RESOLUTION 3D SSFP MR ANGIOGRAPHY FOR ASSESSMENT OF THE THORACIC AORTA IN PREGNANT SUBJECTS WITH FAMILIAL AORTOPATHIES}

L Pickup, *WE Moody, E Plunkett, P Thompson, S Thorne, LE Hudsmith. Department of Cardiology, Queen Elizabeth Hospital Birmingham, Edgbaston, Birmingham, B15 2TH, UK

\subsection{6/heartjnl-2016-309668.19}

Introduction Native magnetic resonance angiography (MRA) is recommended to assess the thoracic aorta during pregnancy, avoiding the risks of ionising radiation and contrast agent administration. This guidance is however, based only on consensus opinion supported by limited case reports (level of evidence C).

Aim To evaluate the feasibility of performing native 3D steadystate free-precession (SSFP) MRA in pregnant subjects with inherited aortopathy to guide timing and mode of delivery.
Methods Prospective patients $(\mathrm{n}=15)$ with known thoracic aortic disease underwent native 3D-SSFP MRA at 1.5T (Avanto, Siemens) with high isotropic spatial resolution $\left(1.3 \times 1.3 \times 1.3 \mathrm{~mm}^{3}\right)$ using a modified ECG-triggered sequence orientated in a sagittal-oblique plane, aligned along the aortic arch, with a respiratory navigator at the diaphragmatic level (slice thickness $2.00 \mathrm{~mm}$; TR/TE: $274 / 1.5 \mathrm{~ms}$ ). Subjects were imaged during "free breathing" adopting a "left uterine displacement" position, with a wedge under the right buttock, offloading the gravid uterus from the inferior vena cava. Aortic dimensions were measured at 7 levels by two independent blinded observers after an assessment of image quality (score 0-3: $0=$ poor, $3=$ excellent).

Results Native 3D-MRA was successfully acquired in all subjects during the mid-trimester $(20.6 \pm 4.9 \mathrm{wk})$. Subject characteristics and pregnancy outcomes are available in Table 1 . Image quality was deemed excellent in $87 \%$ after a mean acquisition time of $3.1 \pm 1.5 \mathrm{~min}$. Figure 1 provides a typical example of a $3 \mathrm{D}$ MRA image. There was a high level of agreement for aortic measurements, with low intra- and inter-observer variability (ICC ranges; 0.95-0.99 and 0.92-0.96, respectively). All pregnancies reached term $(\geq 37 / 40)$ with a mean gestation at delivery of $38.0 \pm 0.5 \mathrm{wk}$. Imaging surveillance in combination with satisfactory haemodynamic status permitted vaginal delivery in $60 \%$. 